\title{
Magnetic phase diagram of dense holographic multiquarks in the quark-gluon plasma
}

\author{
Piyabut Burikham \\ Theoretical High-Energy Physics and Cosmology Group, Department of Physics, \\ Faculty of Science, Chulalongkorn University, \\ Bangkok 10330, Thailand \\ Thailand Center of Excellence in Physics, CHE, Ministry of Education, \\ Bangkok 10400, Thailand \\ Department of Physics, CERN Theory Division, \\ CH-1211 Geneva 23, Switzerland \\ E-mail: piyabut@gmail.com
}

Abstract: We study phase diagram of the dense holographic gauge matter in the SakaiSugimoto model in the presence of the magnetic field above the deconfinement temperature. Even above the deconfinement, quarks could form colour bound states through the remaining strong interaction if the density is large. We demonstrate that in the presence of the magnetic field for a sufficiently large baryon density, the multiquark-pion gradient (MQ$\nabla \varphi$ ) phase is more thermodynamically preferred than the chiral-symmetric quark-gluon plasma. The phase diagrams between the holographic multiquark and the chiral-symmetric quark-gluon plasma phase are obtained at finite temperature and magnetic field. In the mixed MQ- $\nabla \varphi$ phase, the pion gradient induced by the external magnetic field is found to be a linear response for small and moderate field strengths. Its population ratio decreases as the density is raised and thus the multiquarks dominate the phase. Temperature dependence of the baryon chemical potential, the free energy and the linear pion gradient response of the multiquark phase are well approximated by a simple analytic function $\sqrt{1-\frac{T^{6}}{T_{0}^{6}}}$ inherited from the metric of the holographic background.

Keywords: Holography and quark-gluon plasmas, Brane Dynamics in Gauge Theories, D-branes

ARXIV EPRINT: 1103.4379 


\section{Contents}

1 Introduction $\quad 1$

2 Holographic setup of the magnetized multiquark phase 2

$\begin{array}{lll}3 & \text { Magnetic phase diagram of the dense nuclear phase } & 6\end{array}$

4 Conclusion $\quad 12$

A Force condition of the multiquark configuration 12

\section{Introduction}

Discovery of the AdS/CFT correspondence [1] and the generalization in terms of the holographic principle have provided us with alternative theoretical methods to explore the physics of strongly coupled gauge matter. Holographic models have been constructed to mimic behaviour of the strongly coupled gauge matter in various situations. The SakaiSugimoto (SS) model $[2,3]$ is a holographic model which contains chiral fermions in the fundamental representation of $U\left(N_{c}\right)$. Its low energy limit is the closest holographic model of the QCD so far. It can also accommodate distinctively the chiral symmetry restoration and the deconfinement phase transition in the non-antipodal case [4]. It provides interesting possibility of the existence of the exotic nuclear phase where quarks and gluons are deconfined but the chiral symmetry is still broken.

In the SS model, there are two background metrics describing a confined and a deconfined phase. The deconfined phase corresponds to the background metric with a black hole horizon. The Hawking temperature of the black hole is identified with the temperature of the dual "QCD" matter. When gluons are deconfined, the thermodynamical phase of the nuclear matter can be categorized into 3 phases, the vacuum phase, the chirally broken phase and the chiral-symmetric phase. In the deconfined phase, the interaction between quarks and gluons become the screened Coulomb potential. If the coupling is still strong, bound states of quarks could form (see ref. [5-10] for multiquark related studies). The phase diagram of the holographic nuclear matter in the SS model is studied in details in ref. [11] and extended to include multiquarks with colour charges in ref. [10]. It has certain similarity to the conventional QCD phase diagram speculated from other approaches e.g. the existence of critical temperature line above which chiral symmetry is restored. The phase diagram also shows the thermodynamic preference of the multiquark phase with broken chiral symmetry for moderate temperature in the situation when the density is sufficiently large. As an implication, it is thus highly likely that matters in the core of neutron stars are compressed into the multiquark nuclear phase. A thorough investigation on the 
multiquark star suggests higher mass limits of the neutron stars if they have multiquark cores [12].

When the magnetic field is turned on, the phase structure becomes more complicated. Magnetic field induces the pion gradient or a domain wall as a response of the chiral condensate of the chirally broken phase [13]. In the confined phase, this is distinctive [14]. However, it is demonstrated in ref. [15] that the pion gradient is subdominant to the contribution from the multiquarks in the chirally broken deconfined phase. It was also shown in ref. [15] that for sufficiently large density, the multiquark phase is more thermodynamically preferred than the chiral-symmetric quark-gluon plasma for small and moderate magnetic field strengths. Therefore it is interesting to explore the phase diagram of the deconfined nuclear matter in the presence of the external magnetic field. We establish two phase diagrams between the chirally broken multiquark $(\chi \mathrm{SB})$ and the chiral-symmetric quarkgluon plasma ( $\chi \mathrm{S}-\mathrm{QGP})$, one at fixed temperature, $T=0.10$, and another at fixed field, $B=0.20$. The magnetic phase diagram of the similar model for zero baryon density is investigated in ref. [16]. The phase diagram at finite density without instanton source is explored in ref. [17] with the approximation $f(u) \simeq 1$. We found that in the presence of the instanton for $T \gtrsim 0.10$, this approximation is no longer valid.

Our main results demonstrate that for a given magnetic field and moderate temperature, the most preferred nuclear phase in the SS holographic model is the multiquark-pion gradient $(\mathrm{MQ}-\nabla \varphi)$ phase provided that the density is sufficiently large. We also study the temperature dependence of the baryon chemical potential, the free energy, and the linear response of the pion gradient of the mixed MQ- $\nabla \varphi$ phase and show that they inherit the temperature dependence mostly from the SS background.

Extremely strong magnetic fields could have been produced in many situations. The Higgs mechanism in the cosmological electroweak phase transition could create enormous magnetic fields in the region between two different domains with different Higgs vacuum expectation values [18] which could play vital role in the phase transitions of the nuclear soup at later times. At the hadron and heavy ion colliders, colliding energetic charged particles could produce exceptional strong magnetic field locally. The local magnetic fields produced at RHIC and LHC are estimated to be in the order of $10^{14-15}$ Tesla [19]. On the astrophysical scale, certain types of neutron stars called the magnetars could produce magnetic fields as strong as $10^{10}$ Tesla [20].

This article is organized as the following. In section 2, the setup of the deconfined SS model with additional baryon vertex and string sources are discussed. Main results are elaborated in section 3. Section 4 concludes the article.

\section{Holographic setup of the magnetized multiquark phase}

The setup we will use is the same as in ref. [15], the Sakai-Sugomoto model with additional baryon vertex and strings (baryon vertex is introduced in ref. [21, 22]). Starting from a 10 dimensional type IIA string theory with one dimension compactified into a circle which we will label $x^{4}$. Two stacks of D8-branes and $\overline{\mathrm{D} 8}$-branes are then located at distance $L$ from each other in the $x^{4}$ direction at the boundary. This separation will be fixed 
at the boundary and it will play the role of the fundamental scale of our holographic model. Open-string excitations with one end on the D8 and $\overline{\mathrm{D} 8}$ will represent quarks with different chiralities. In the background where the $\mathrm{D} 8$ and $\overline{\mathrm{D} 8}$ are parallel, excitations for each chirality are independent and there is a chiral symmetry in the background and at the boundary. For background with connecting D8 and $\overline{\mathrm{D} 8}$, chiral symmetry is broken and there is a chiral condensate. When the energy of the connecting configuration is minimal and there is no extra sources, we define the corresponding boundary gauge matter to be in a vacuum phase.

Since the partition function of the string theory in the bulk is conjectured to be equal to the partition function of the gauge theory on the boundary, the free energy of the boundary gauge matter is equivalent to the superstring action in the bulk (modulo a periodicity factor) [23]. We turn on non-normalizable modes of the gauge field $a_{3}^{V}, a_{1}^{A}, a_{0}^{V}$ (defined in units of $R_{D 4} / 2 \pi \alpha^{\prime}$ ) in the D8-branes and identify them with the vector potential of the magnetic field, $B$ (defined in units of $1 / 2 \pi \alpha^{\prime}$ ), the gradient of the chiral condensate, $\nabla \varphi$, and the baryon chemical potential, $\mu$, at the boundary respectively. These curious holographic correspondence between the branes' fields and the thermodynamical quantities of the gauge matter at the boundary allows us to study physics of the strongly coupled non-Abelian gauge matter at finite density in the presence of the external magnetic field. Electric field can also be added using other components of the gauge field on the D8branes [16, 24] but we will not consider such cases here.

The background spacetime of the Sakai-Sugimoto model is in the form

$$
\begin{aligned}
& d s^{2}=\left(\frac{u}{R_{D 4}}\right)^{3 / 2}\left(f(u) d t^{2}+\delta_{i j} d x^{i} d x^{j}+d x_{4}^{2}\right)+\left(\frac{R_{D 4}}{u}\right)^{3 / 2}\left(u^{2} d \Omega_{4}^{2}+\frac{d u^{2}}{f(u)}\right) \\
& F_{(4)}=\frac{2 \pi N}{V_{4}} \epsilon_{4}, \quad e^{\phi}=g_{s}\left(\frac{u}{R_{D 4}}\right)^{3 / 4}, \quad R_{D 4}^{3} \equiv \pi g_{s} N_{c} l_{s}^{3},
\end{aligned}
$$

where $f(u) \equiv 1-u_{T}^{3} / u^{3}, u_{T}=16 \pi^{2} R_{\mathrm{D} 4}^{3} T^{2} / 9 . V_{4}$ is the volume of the unit four-sphere $\Omega_{4}$ and $\epsilon_{4}$ represents the volume 4 -form. $l_{s}$ and $g_{s}$ are the string length scale and the string coupling respectively. $R$ is the compactified radius of the $x^{4}$ coordinate. This radius is different from the curvature $R_{D 4}$ of the background in general. The dilaton field is denoted by $\phi$ which will be eliminated by the function of $u$ as stated above.

The direction of the magnetic field is chosen so that the vector potential is

$$
a_{3}^{V}=B x_{2} .
$$

The baryon chemical potential $\mu$ of the corresponding gauge matter is identified with the non-normalizable mode of the DBI gauge field at the boundary by

$$
\mu=a_{0}^{V}(u \rightarrow \infty)
$$

The five-dimensional Chern-Simon term of the D8-branes generates another axial part of the $U(1), a_{1}^{A}$, by coupling it with $B$ and $a_{0}^{V}$. In this way, the external magnetic field induces the axial current $j_{A}$ associated with the axial field $a_{1}^{A}$. The non-normalizable mode of this field at the boundary corresponds to the response of the chiral condensate to the 
magnetic field which we call the pion gradient, $\nabla \varphi$. External field causes the condensate to form a domain wall which can be characterized by the gradient of the condensate with respect to the direction of the applied field. The pion gradient also acts as a source of the baryon density in our gauge matter.

Additional sources of the baryon density and the baryon chemical potential can be added to the configuration in the form of the baryon vertex and strings. The vertex appears as an instanton at the tip $u_{c}$ of the brane configuration and the strings hang down from the vertex to the horizon $u_{T}[10,11]$.

$$
\begin{aligned}
S_{\text {source }} & =\mathcal{N} d\left[\frac{1}{3} u_{c} \sqrt{f\left(u_{c}\right)}+n_{s}\left(u_{c}-u_{T}\right)\right], \\
& =\mathcal{N} d \mu_{\text {source }}
\end{aligned}
$$

where $n_{s}=k_{r} / N_{c}$ is the number of radial strings in the unit of $1 / N_{c}$. Since the radial strings could merge with strings from other multiquark and generate a binding potential between the multiquarks, this number therefore represents the colour charges of the multiquark in the deconfined phase. It is interesting to note that when there is only string source representing quark matter, the quark matter becomes thermodynamically unstable under density fluctuations [11]. However, adding baryon vertex together with the strings makes the multiquark configuration stable under the density fluctuations [10]. The multiquark phase is even more thermodynamically preferred than the $\chi \mathrm{S}$-QGP when the density is sufficiently large and the temperature is not too high.

With this setup, then the DBI and the Chern-Simon actions of the D8-branes configuration can be calculated to be

$$
\begin{aligned}
& S_{D 8}=\mathcal{N} \int_{u_{c}}^{\infty} d u u^{5 / 2} \sqrt{1+\frac{B^{2}}{u^{3}}} \sqrt{1+f(u)\left(a_{1}^{\prime A}\right)^{2}-\left(a_{0}^{\prime V}\right)^{2}+f(u) u^{3} x_{4}^{\prime 2}}, \\
& S_{C S}=-\frac{3}{2} \mathcal{N} \int_{u_{c}}^{\infty} d u\left(\partial_{2} a_{3}^{V} a_{0}^{V} a_{1}^{A \prime}-\partial_{2} a_{3}^{V} a_{0}^{V \prime} a_{1}^{A}\right),
\end{aligned}
$$

where $\mathcal{N}=N R_{D 4}^{2} /\left(6 \pi^{2}\left(2 \pi \alpha^{\prime}\right)^{3}\right)$ defines the brane tension. The factor $3 / 2$ in the ChernSimon action fixes the surface effect of the region with uniform magnetic field as explained in ref. [14]. We have to add extra surface terms to preserve the gauge invariance since the gauge transformation does not vanish at the boundary in this case.

We can write down the equations of motion with respect to each gauge field $a_{0}^{V}, a_{1}^{A}$ as

$$
\begin{aligned}
& \frac{\sqrt{u^{5}+B^{2} u^{2}} f(u) a_{1}^{\prime A}}{\sqrt{1+f(u)\left(a_{1}^{\prime A}\right)^{2}-\left(a_{0}^{\prime V}\right)^{2}+f(u) u^{3} x_{4}^{\prime 2}}}=j_{A}-\frac{3}{2} B \mu+3 B a_{0}^{V}, \\
& \frac{\sqrt{u^{5}+B^{2} u^{2}} a_{0}^{\prime V}}{\sqrt{1+f(u)\left(a_{1}^{\prime A}\right)^{2}-\left(a_{0}^{\prime V}\right)^{2}+f(u) u^{3} x_{4}^{\prime 2}}}=d-\frac{3}{2} B a_{1}^{A}(\infty)+3 B a_{1}^{A} .
\end{aligned}
$$

$d, j_{A}$ are the corresponding density and current density of the dual gauge matter at the boundary of the background $(u \rightarrow \infty)$ given by

$$
\begin{aligned}
j^{\mu}(x, u \rightarrow \infty) & \left.\equiv \frac{\delta S_{e o m}}{\delta A_{\mu}}\right|_{u \rightarrow \infty} \\
& \equiv\left(d, \overrightarrow{j_{A}}\right) .
\end{aligned}
$$


In terms of the gauge fields, they are

$$
\begin{gathered}
d=\left.\frac{\sqrt{u^{5}+B^{2} u^{2}} a_{0}^{\prime V}}{\sqrt{1+f(u)\left(a_{1}^{\prime A}\right)^{2}-\left(a_{0}^{\prime V}\right)^{2}+f(u) u^{3} x_{4}^{\prime 2}}}\right|_{\infty}-\frac{3}{2} B a_{1}^{A}(\infty), \\
j_{A}=\left.\frac{\sqrt{u^{5}+B^{2} u^{2}} f(u) a_{1}^{\prime A}}{\sqrt{1+f(u)\left(a_{1}^{\prime A}\right)^{2}-\left(a_{0}^{\prime V}\right)^{2}+f(u) u^{3} x_{4}^{\prime 2}}}\right|_{\infty}-\frac{3}{2} B \mu .
\end{gathered}
$$

In order to solve these equations, we need to specify the boundary conditions. Due to the holographic nature of the background spacetime, the boundary conditions correspond to physical requirement we impose to the gauge matter. If we want to address chirally broken phase of the gauge matter, we will take $a_{1}^{A}(\infty) \equiv \nabla \varphi$ to be an order parameter of the chiral symmetry breaking (also a response to the external magnetic field) and minimize the action with respect to it. This results in setting $j_{A}=0$. On the other hand, if we want to study the chiral-symmetric gauge matter (or chiral-symmetric quark-gluon plasma for $N_{c}=3$ case), $x_{4}^{\prime}$ and $a_{1}^{A}(\infty)$ will be set to zero. For vacuum phase, $a_{0}^{V}, a_{1}^{A}$ and $d, j_{A}$ will be set to zero.

In any cases, since the total action does not depend on $x_{4}(u)$ explicitly, the constant of motion gives

$$
\left(x_{4}^{\prime}(u)\right)^{2}=\frac{1}{u^{3} f(u)}\left[\frac{u^{3}\left[f(u)\left(C(u)+D(u)^{2}\right)-\left(j_{A}-\frac{3}{2} B \mu+3 B a_{0}^{V}\right)^{2}\right]}{F^{2}}-1\right]^{-1},
$$

where

$$
F=\frac{u_{c}^{3} \sqrt{f\left(u_{c}\right)} \sqrt{f\left(u_{c}\right)\left(C\left(u_{c}\right)+D\left(u_{c}\right)^{2}\right)-\left(j_{A}-\frac{3}{2} B \mu+3 B a_{0}^{V}\left(u_{c}\right)\right)^{2}} x_{4}^{\prime}\left(u_{c}\right)}{\sqrt{1+f\left(u_{c}\right) u_{c}^{3} x_{4}^{\prime 2}\left(u_{c}\right)}}
$$

and $C(u) \equiv u^{5}+B^{2} u^{2}, D(u) \equiv d+3 B a_{1}^{A}(u)-3 B \nabla \varphi / 2$. The constant of motion with respect to $x_{4}(u)$ relates the slope $x_{4}^{\prime}$ at arbitrary $u$ to the value at $u_{c}$,

$$
\left(x_{4}^{\prime}\left(u_{c}\right)\right)^{2}=\frac{1}{f_{c} u_{c}^{3}}\left[\frac{9}{d^{2}} \frac{\left(f_{c}\left(C_{c}+D_{c}^{2}\right)-\left(j_{A}-\frac{3}{2} B \mu+3 B a_{0}^{V}\left(u_{c}\right)\right)^{2}\right)}{\left(1+\frac{1}{2}\left(\frac{u_{T}}{u_{c}}\right)^{3}+3 n_{s} \sqrt{f_{c}}\right)^{2}}-1\right] .
$$

The calculation of $x_{4}^{\prime}\left(u_{c}\right)$ is described in the appendix as a result from the equilibrium and scale fixing condition

$$
L_{0}=2 \int_{u_{c}}^{\infty} x_{4}^{\prime}(u) d u=1
$$

The equations of motion eq. (2.8), (2.9) can be solved numerically under the constraint (2.16). The value of $\mu, \nabla \varphi, u_{c}$ and the initial values of $a_{0}^{V}\left(u_{c}\right), a_{1}^{A}\left(u_{c}\right)$ are chosen so that $a_{0}^{V}(\infty)=\mu, a_{1}^{A}(\infty)=\nabla \varphi$ and $L_{0}=1$ are satisfied simultaneously. Since there are 3 conditions to be satisfied in finding the physical solutions numerically, we need the use the shooting algorithm for 3 targets at once. 


\section{Magnetic phase diagram of the dense nuclear phase}

Generically, the action (2.6) and (2.7) are divergent from the $u \rightarrow \infty$ limit of the integration and we need to regulate it using the action of the vacuum which is also divergent. The contribution from the region $u \rightarrow \infty$ is divergent even when the magnetic field is turned off and it is intrinsic to the DBI action in this background. The divergence can be understood as the infinite zero-point energy of the system and thus could be systematically removed by regularisation.

Therefore the regulated free energy is given by

$$
\mathcal{F}_{\mathrm{E}}=\Omega(\mu, B)+\mu d
$$

where $\Omega(\mu, B)=S\left[a_{0}(u), a_{1}(u)\right]($ e.o.m. $)-S[$ magnetized vacuum]. Note that we need to Legendre transform the DBI and the Chern-Simon action to obtain the bulk action as a function of the non-normalizable modes $a_{0}^{V}, a_{1}^{A}$ in order to identify it with the free energy of the gauge matter at the boundary. In terms of the free energy at the boundary, this is equivalent to the Legendre transform of the grand canonical with respect to $\mu$ and $d$.

We can calculate the total action satisfying the equation of motion $S\left[a_{0}(u), a_{1}(u)\right]($ e.o.m. $)=S_{D 8}+S_{C S}$ to be

$$
\begin{aligned}
S_{D 8} & =\mathcal{N} \int_{u_{c}}^{\infty} d u C(u) \sqrt{\frac{f(u)\left(1+f(u) u^{3} x_{4}^{\prime 2}\right)}{f(u)\left(C(u)+D(u)^{2}\right)-\left(j_{A}-\frac{3}{2} B \mu+3 B a_{0}^{V}\right)^{2}}}, \\
S_{C S} & =-\mathcal{N} \frac{3}{2} B \int_{u_{c}}^{\infty} d u \frac{\left(a_{0}^{V}\left(j_{A}-\frac{3}{2} B \mu+3 B a_{0}^{V}\right)-f(u) D(u) a_{1}^{A}\right) \sqrt{\frac{1}{f(u)}+u^{3} x_{4}^{\prime 2}}}{\sqrt{f(u)\left(C(u)+D(u)^{2}\right)-\left(j_{A}-\frac{3}{2} B \mu+3 B a_{0}^{V}\right)^{2}}} .
\end{aligned}
$$

The three nuclear phases above the deconfinement temperature are governed by the same equations of motion, each with specific boundary conditions as the following,

magnetized vacuum phase: $a_{0}^{V}, a_{1}^{A}=0 ; d, j_{A}=0$,

multiquark-pion gradient phase: $a_{0}^{V}\left(u_{c}\right)=\mu_{\text {source }}, a_{1}^{A}\left(u_{c}\right)=0, a_{1}^{A}(\infty)=\nabla \varphi, j_{A}=0$,

$\chi$ S-QGP phase: $x_{4}^{\prime}(u)=0, a_{0}^{V}\left(u_{c}=u_{T}\right)=0, a_{1}^{A}(\infty)=0, j_{A}=\frac{3}{2} B \mu$.

We will demonstrate later that in the mixed phase, the pion gradient is generically dominated by the multiquark when the chiral symmetry is broken. In ref. [15], it is shown that the pure pion gradient phase is always less preferred thermodynamically than the mixed phase of $\mathrm{MQ}-\nabla \varphi$. It is interesting to note that for the pure pion gradient phase, a large magnetic field is required in order to stabilize the generated domain wall [13]. This critical field is determined by the mass of the pion in the condensate, $B_{c r i t} \sim m_{\pi}^{2} / e$. In ref. [15], this critical behaviour is confirmed in the holographic SS model (the zero-temperature situation is studied in ref. [25]). More investigation of the pure pion gradient phase in the holographic model should be conducted especially when the field is large since the distinctive feature of physics from the DBI action becomes apparent in this limit. We will leave this task for future work and focus our attention to the mixed MQ- $\nabla \varphi$ phase in this article. 


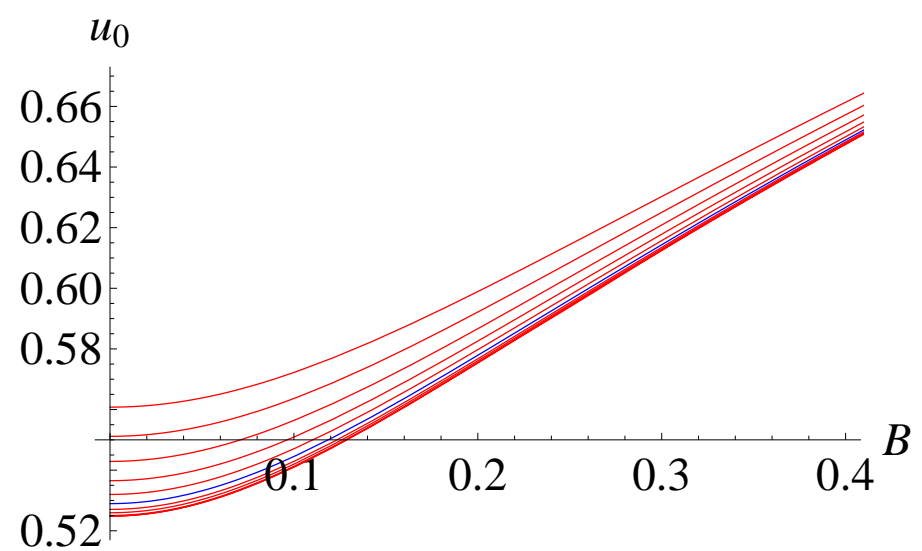

Figure 1. The position $u_{0}$ of the connected D8- $\overline{\mathrm{D} 8}$ vacuum configuration as a function of $B$ for $T=0.02-0.15$. The upper lines have higher temperatures.

The action of the magnetized vacuum when we set $a_{0}^{V}, a_{1}^{A}=0$ and $d, j_{A}=0$ is

$$
S[\text { magnetized vacuum }]=\left.\int_{u_{0}}^{\infty} \sqrt{C(u)\left(1+f(u) u^{3} x_{4}^{\prime 2}\right)}\right|_{v a c} d u
$$

where

$$
\left.x_{4}^{\prime}(u)\right|_{\text {vac }}=\frac{1}{\sqrt{f(u) u^{3}\left(\frac{f(u) u^{3} C(u)}{f\left(u_{0}\right) u_{0}^{3} C\left(u_{0}\right)}-1\right)}} .
$$

The position $u_{0}$ where $x_{4}^{\prime} \rightarrow \infty$ is the tip of the brane configuration of the magnetized vacuum. It increases slightly with temperature as is shown in figure 1 . The difference between each temperature decreases as the magnetic field gets larger and all curves converge to the same saturated value $u_{0}=1.23$ in the large field limit.

We can study the temperature dependence of the magnetized multiquark nuclear matter by considering its baryon chemical potential and the free energy as shown in figure 2 . Both the chemical potential and the free energy decrease steadily as the temperature rises, regardless of the magnetic field. This is originated from the temperature dependence of $f(u)=1-\frac{u_{T}^{3}}{u^{3}}$ of the SS background in the deconfined phase. The temperature dependence could be fit very closely with the function $\sqrt{1-\left(\frac{T}{T_{0}}\right)^{6}}$ as the following

$$
\begin{aligned}
& \mu=\mu_{0}(d, B) \sqrt{1-\left(\frac{T}{T_{0}}\right)^{6}}, \\
& F=F_{0}(d, B) \sqrt{1-\left(\frac{T}{T_{0}}\right)^{6}} .
\end{aligned}
$$

where for $d=1, B=0.10 ; \mu_{0}=1.1849, F_{0}=0.7976$ respectively. For the baryon chemical potential (free energy), the best-fit value of $T_{0}$ is 0.269 (0.233). The fittings are shown in figure 3. This could be explained by noting that the regulated free energy is given by 


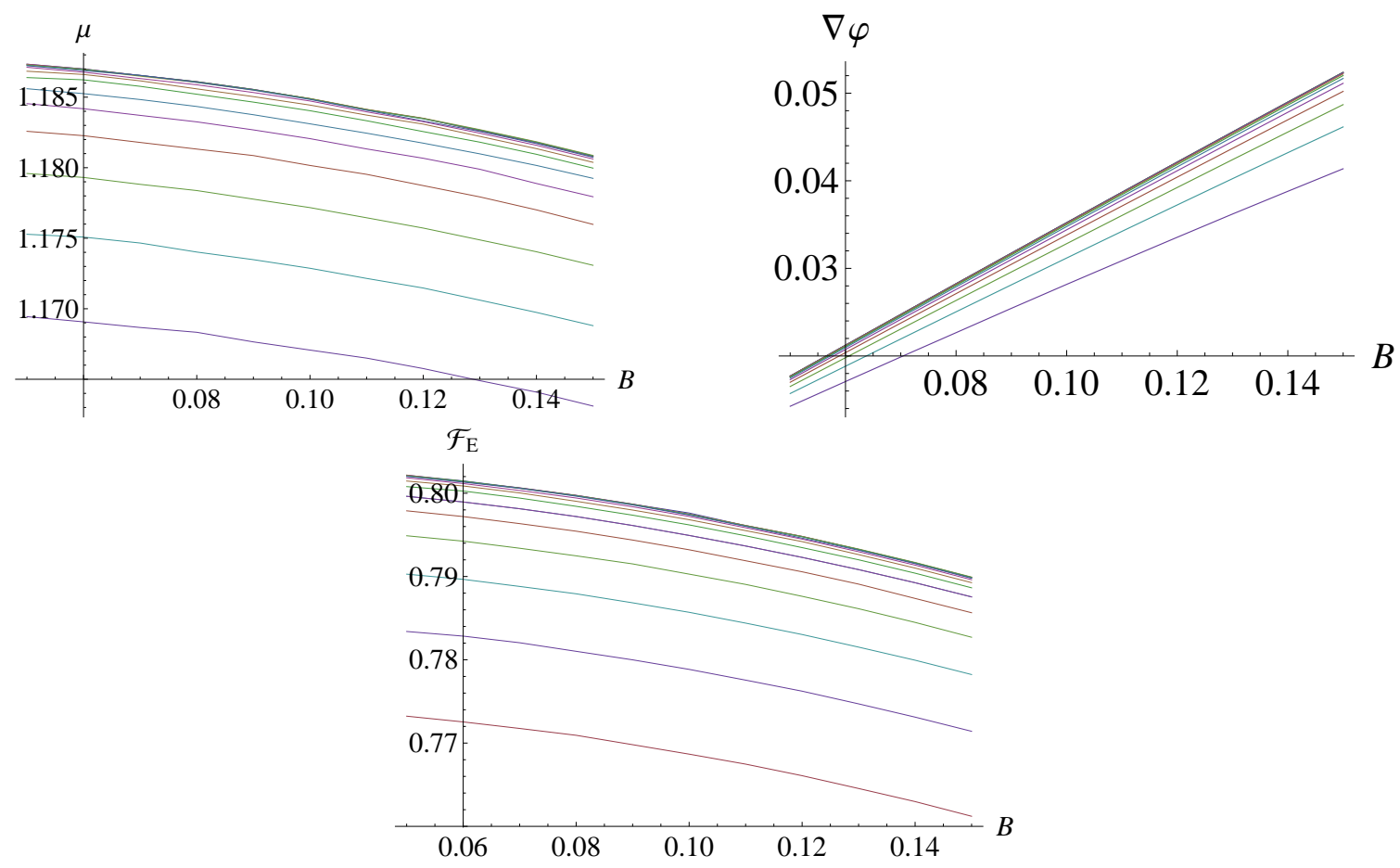

Figure 2. The chemical potential (a), the pion gradient (b), and the free energy (c) of the multiquark phase with baryon density $d=1$ as a function of $B$ for temperature $T=0.02-0.15$. The lower curves represent multiquark at higher temperatures.

$\mu d+\Omega(\mu, B)$. The contribution from the first term is dominant therefore the free energy has almost the same temperature dependence as the chemical potential. However, there is a minor contribution from $S_{\mathrm{D} 8}+S_{\mathrm{CS}}$ containing $f\left(u_{c}\right)=1-\frac{u_{T}^{3}}{u_{c}^{3}}$ which for small temperature fractions modifies the temperature function in the following manner,

$$
C_{1} \sqrt{1-\frac{T^{6}}{T_{1}^{6}}}+C_{2} \sqrt{1-\frac{T^{6}}{T_{2}^{6}}} \simeq C_{0} \sqrt{1-\frac{T^{6}}{T_{0}^{6}}},
$$

where $C_{1,2}$ are some arbitrary constants and $C_{0}, T_{0}$ are given by

$$
\begin{aligned}
C_{0} & =C_{1}+C_{2}, \\
\frac{1}{T_{0}^{6}} & =\frac{1}{C_{1}+C_{2}}\left(\frac{C_{1}}{T_{1}^{6}}+\frac{C_{2}}{T_{2}^{6}}\right) .
\end{aligned}
$$

It should be noted from figure 3 that the temperature dependence is significant for $T \gtrsim 0.10$ and the approximation $f(u) \simeq 1$ is not accurate for temperature in this range. The characteristic temperatures we found here are consistent with the phase diagram of the multiquark in figure 7 .

In the multiquark phase when the magnetic field is turned on, the pion gradient is induced by the field in addition to the multiquark. The multiquark phase thus contained the mixed content of multiquarks and the pion gradient. For moderate fields (not too large), the response is linear $\nabla \varphi \propto B$. In contrast to the case of pure pion gradient phase, 

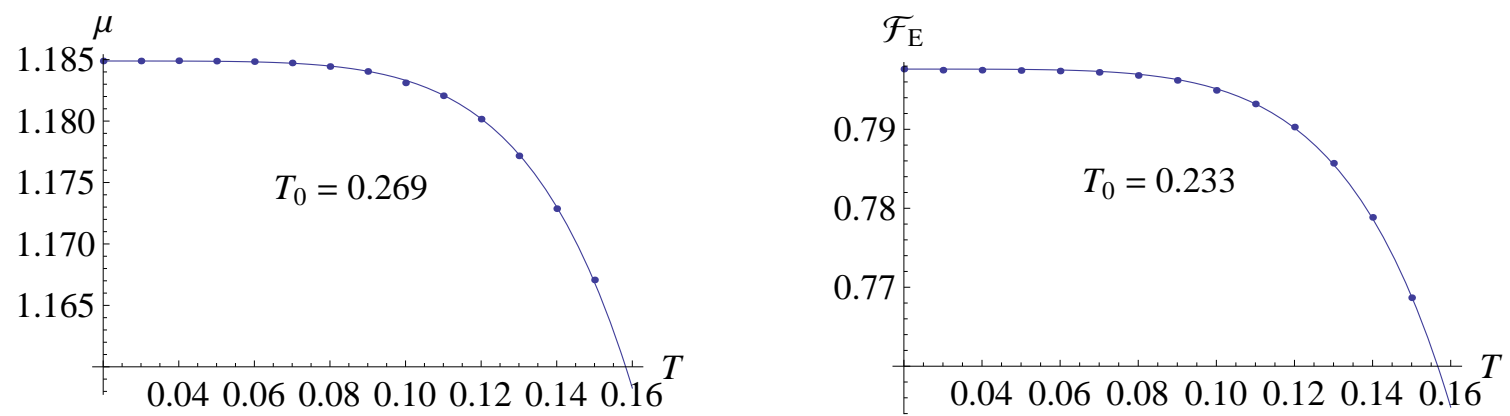

Figure 3. For $d=1, B=0.10$,(a) the baryon chemical potential as a function of $T$, the best-fit curve is in the form $\mu_{0} \sqrt{1-\left(\frac{T}{T_{0}}\right)^{6}}$ with $\mu_{0}=1.1849, T_{0}=0.269 ;(\mathrm{b})$ the free energy as a function of $T$, the best-fit curve is in the form $F_{0} \sqrt{1-\left(\frac{T}{T_{0}}\right)^{6}}$ with $F_{0}=0.7976, T_{0}=0.233$. Other curves within the range $B=0.05-0.15$ can also be fitted well with the same $T_{0}$.

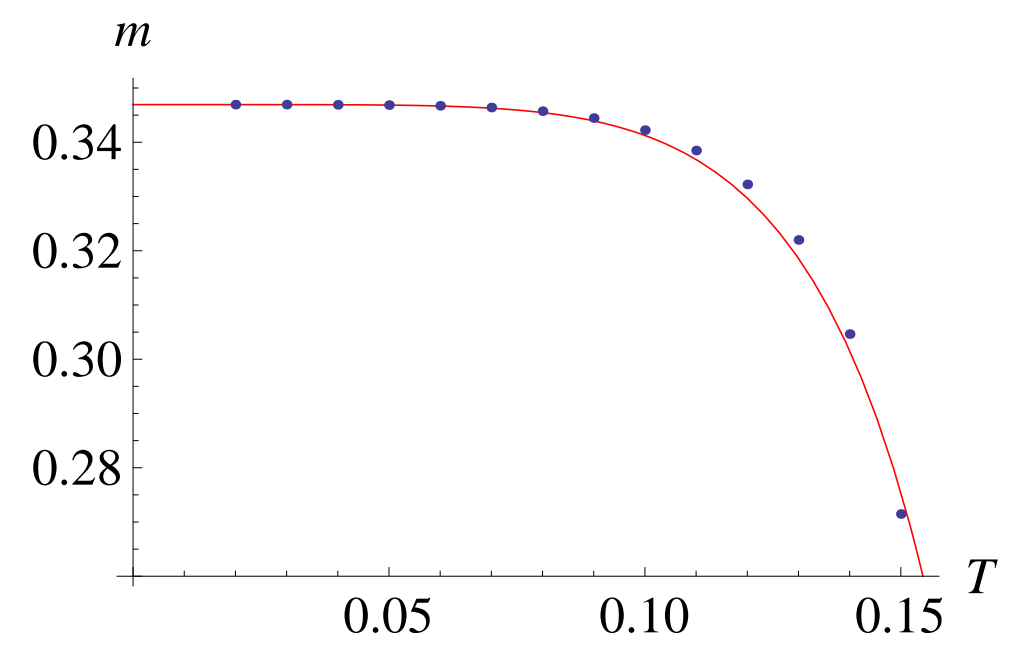

Figure 4. The linear response or slope of the linear function between the pion gradient and the magnetic field as a function of the temperature for the range $B=0.05-0.15$ and density $d=1$. The red line is the best-fit curve in the form $m_{0} \sqrt{1-\left(\frac{T}{T_{0}}\right)^{6}}$ with $m_{0}=0.347, T_{0}=0.177$.

the domain wall in the mixed $\mathrm{MQ}-\nabla \varphi$ phase is stable among the surrounding multiquarks even for small field. The critical magnetic field to stabilize the domain wall in the case of pure pion gradient is not required in the mixed phase.

Figure 2 (b) shows a linear relation between the pion gradient and the magnetic field which is valid up to moderate fields. For $d=1$, we found that the slope, $m$ (or the linear response), of this linear function depends on the temperature approximately as $m=m_{0} \sqrt{1-\left(\frac{T}{T_{0}}\right)^{6}}$, and

$$
\nabla \varphi \simeq B m_{0} \sqrt{1-\left(\frac{T}{T_{0}}\right)^{6}},
$$

where $m_{0}=0.347, T_{0}=0.177$. The curve fitting is shown in figure 4 . The density depen- 

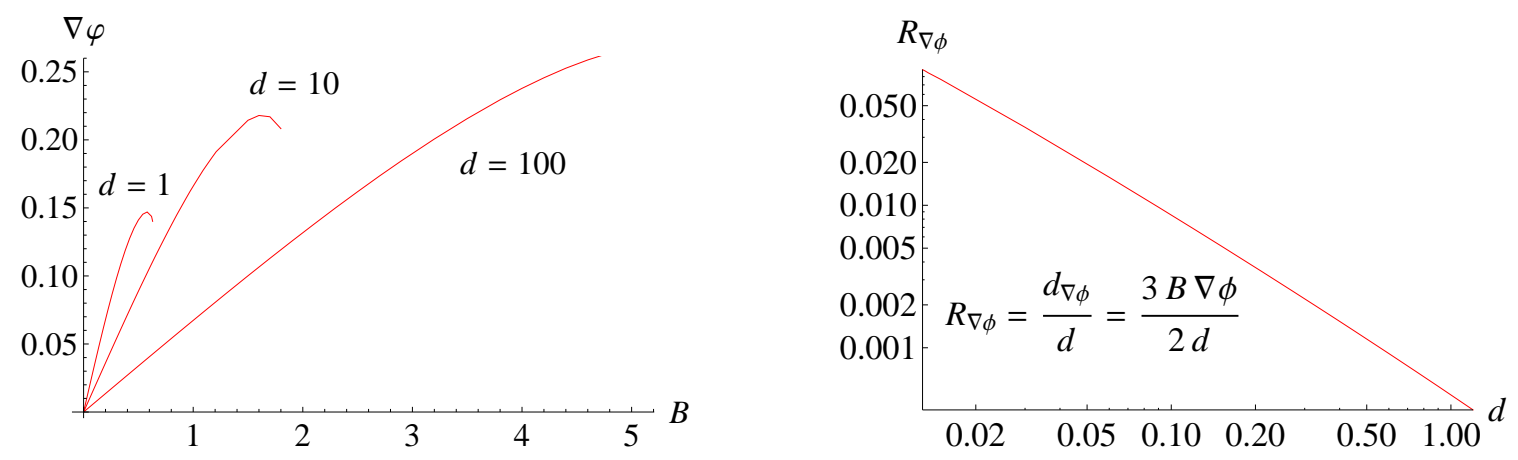

Figure 5. (a) The pion gradient as a function of $B$ for density $d=1,10,100$ at $T=0.10$. (b) The density ratio of the pion gradient with respect to the total baryon density of the multiquark phase at $B=0.10, T=0.10$ in the double-log scale.

dence is encoded in $m_{0}=m_{0}(d), T_{0}=T_{0}(d)$. As the density increases, the slope of the linear response of the pion gradient becomes smaller as is shown in figure 5 . The ratio of the pion gradient density and the total baryonic density $R_{\nabla \varphi} \equiv d_{\nabla \varphi} / d=3 B \nabla \varphi / 2 d[14]$ for $B=$ $0.10, T=0.10$ is plotted in the log-scale in figure $5(\mathrm{~b})$. It could be well approximated by

$$
\begin{aligned}
R_{\nabla \varphi} & \simeq \text { (const. }) d^{-6 / 5} \\
& \simeq \frac{3 B^{2} m_{0}}{2 d} \sqrt{1-\left(\frac{T}{T_{0}}\right)^{6}},
\end{aligned}
$$

from eq. (3.10). This implies that the multiquark states are more preferred than the pion gradient in the presence of the magnetic field, the denser the nuclear matter, the more stable the multiquarks become and the lesser the population of the pion gradient.

Finally we compare the free energy of the $\mathrm{MQ}-\nabla \varphi$ phase and the chiral-symmetric quark-gluon plasma phase. For high density, $d=100$, this is shown in figure 6 . For a given density, the multiquark phase is more thermodynamically preferred than the $\chi \mathrm{S}$-QGP for small and moderate fields. As the magnetic field gets larger, the $\chi \mathrm{S}-\mathrm{QGP}$ becomes more thermodynamically preferred. When the field becomes very strong, the transition into the lowest Landau level finally occurs [26]. For a fixed density, increasing magnetic field inevitably results in the chiral symmetry restoration. The phase transition between the $\mathrm{MQ}-\nabla \varphi$ and the $\chi \mathrm{S}-\mathrm{QGP}$ is a first order since the free energy is continuous at the transition and the slope has a discontinuity. It implies that the magnetization, $M(d, B)=-\frac{\partial \mathcal{F}_{\mathrm{E}}}{\partial B}$, of the nuclear matter abruptly changes at the transition.

On the other hand, for a fixed field and the moderate temperature, the increase in the baryon density could make the multiquark phase more stable than the $\chi \mathrm{S}-\mathrm{QGP}$. This is shown in the phase diagram in figure 7 . At a given magnetic field, the multiquark phase could become the most preferred magnetized nuclear phase provided that the density is made sufficiently large and the temperature is not too high. In contrast, the effect of the temperature is the most dominant for chiral-symmetry restoration even when the field is turned on. Sufficiently large temperature will induce chiral-symmetry restoration for 

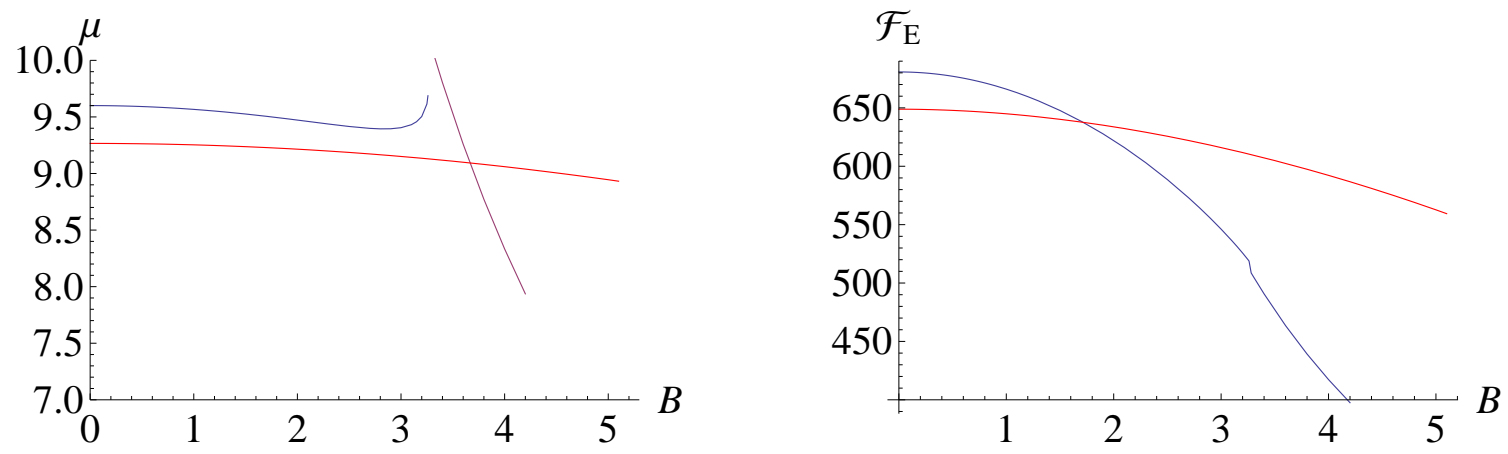

Figure 6. For the dense multiquark with $d=100, T=0.10$, (a) the chemical potential, (b) the free energy as a function of $B$. The multiquark curves in red are compared with the $\chi \mathrm{S}-\mathrm{QGP}$ curves in blue for the chemical potential and the free energy.
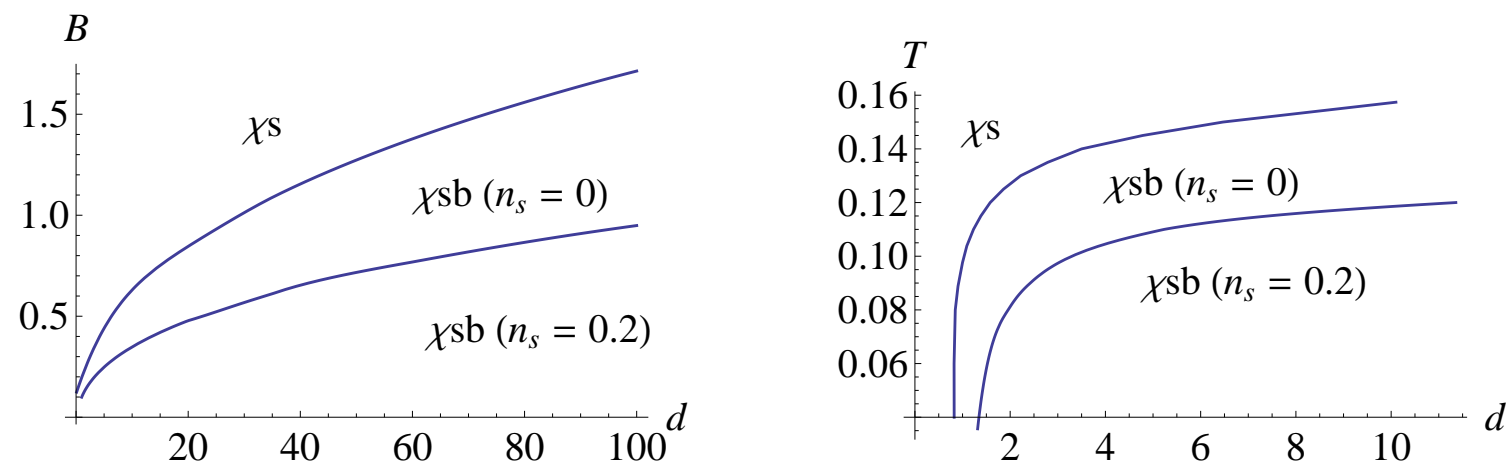

Figure 7. The phase diagram of the dense nuclear phases involving multiquarks when gluons are deconfined for (a) $T=0.10$ and (b) $B=0.20$. The chiral-symmetric quark-gluon plasma and the chirally broken MQ- $\nabla \varphi$ phase are represented by $\chi \mathrm{S}$ and $\chi \mathrm{SB}$ respectively, $n_{s}$ is the number of colour strings in fractions of $1 / N_{c}$.

most densities as is shown the figure 7(b). Nevertheless, theoretically we can always find sufficiently large density above which the multiquark phase is more preferred.

The transition line between the MQ- $\nabla \varphi$ and the $\chi \mathrm{S}$-QGP phases in the $(d, B)$ phase diagram can be approximated by a power-law

$$
B \sim d^{0.438}(0.436)
$$

for the multiquark with $n_{s}=0(0.2)$. This power-law is weaker than the transition line of the $\chi \mathrm{S}$-QGP to the lowest Landau level studied in ref. [26] for the antipodal SS model $\left(B \sim d^{2 / 3}\right)$. The multiquarks with more colour charges $\left(n_{s}>0\right)$ are less preferred thermodynamically but they require higher densities. On the other hand, the transition line in the $(d, T)$ phase diagram is an increasing function of $d$ but weaker than the logarithmic of the density. Nevertheless, theoretically for a fixed $B, T$, we can always find sufficiently large density above which the $\mathrm{MQ}-\nabla \varphi$ phase is preferred. The high density region is actually dominated by the multiquark phase indeed. 


\section{Conclusion}

We explore the properties of the miltiquark-domain wall $(\mathrm{MQ}-\nabla \varphi)$ solution of the SS model above the deconfinement temperature. The temperature dependence of the baryon chemical potential, the pion gradient linear response $(m)$, and the free energy of the MQ$\nabla \varphi$ phase has been studied and fitted with a simple function, $\sqrt{1-\frac{T^{6}}{T_{0}^{6}}}$, inherited from the deconfined SS background. Their characteristic temperatures, $T_{0}$, are different from one another depending on other parameters such as $u_{c}$, the position of the baryon vertex. Remarkably, they do not depend on the field for moderate field strength $B=0.05-0.15$.

For chirally broken deconfined nuclear matter in the presence of the magnetic field, the nuclear matter with finite baryon density and chemical potential could respond to the magnetic field by inducing a pion gradient or a domain wall of the chiral condensate. This pion gradient response is found to be a linear function of the field for moderate fields at any density. However, we demonstrate further that the population ratio of the pion gradient decreases as the density increases. The other sources of the baryon charge namely the multiquarks finally dominate the chirally broken nuclear phase and most of the baryon density is in the form of the multiquark at high density.

Magnetic phase diagram of the dense gauge matter have been explored in the deconfined SS model. At fixed magnetic field and moderate temperature, the $\mathrm{MQ}-\nabla \varphi$ phase are more preferred than the $\chi \mathrm{S}-\mathrm{QGP}$ for the high density region. The transition line in the $(d, B)$ phase diagram at $T=0.10$ can be fitted closely with the power-law $B \sim d^{0.438}(0.436)$ for the multiquark with $n_{s}=0(0.2)$. On the other hand, the transition line in the $(d, T)$ phase diagram is weaker than the logarithmic of the density but nevertheless it is an increasing function with respect to the density. These imply that for sufficiently large density, the chirally broken multiquark phase is the most preferred nuclear phase even in the presence of the external magnetic field.

The situation when density becomes extremely large and being dominant occurs in the core of dense star such as the neutron star. Therefore it is very likely that the core of dense warm star composes primarily of the multiquark nuclear matter even when an enormous magnetic field is present such as in the core of the magnetars. It is possible that a large population of the warm magnetars has multiquark cores. These warm dense objects could be relatively more massive than typical neutron stars.

\section{Acknowledgments}

I would like to thank CERN Theory Division for the warm hospitality during my visit where this work is completed. P.B. is supported in part by the Thailand Research Fund (TRF) and Commission on Higher Education (CHE) under grant RMU5380048 and Thailand Center of Excellence in Physics (ThEP).

\section{A Force condition of the multiquark configuration}

Fixing the characteristic scale $L_{0}$ to 1 for the brane configuration requires balancing three forces in the gravity picture. The D8-brane tension must be in equilibrium with the tidal 
weight of the D4 source and the string tension of the colour strings. The derivation of the $x_{4}^{\prime}\left(u_{c}\right)$ presented here is the same as in ref. [15], it is included for completeness.

We vary the total action with respect to $u_{c}$ to obtain the surface term. Imposing the scale-fixing condition $2 \int_{u_{c}}^{\infty} d u x_{4}^{\prime}(u)=L_{0}=1$, we found that [11]

$$
x_{4}^{\prime}\left(u_{c}\right)=\left(\tilde{L}\left(u_{c}\right)-\frac{\partial S_{\text {source }}}{\partial u_{c}}\right) /\left.\frac{\partial \tilde{S}}{\partial x_{4}^{\prime}}\right|_{u_{c}},
$$

as the condition on $u_{c}$.

We perform the Legendre transformed action with respect to $a_{0}^{V \prime}$ and $a_{1}^{A \prime}$ to obtain

$$
\begin{aligned}
\tilde{S}= & \int_{u_{c}}^{\infty} \tilde{L}\left(x_{4}^{\prime}(u), d\right) d u, \\
= & \mathcal{N} \int_{u_{c}}^{\infty} d u \sqrt{\frac{1}{f(u)}+u^{3} x_{4}^{\prime 2}} \\
& \times \sqrt{f(u)\left(C(u)+D(u)^{2}\right)-\left(j_{A}-\frac{3}{2} B \mu+3 B a_{0}^{V}\right)^{2}},
\end{aligned}
$$

where $C(u) \equiv u^{5}+B^{2} u^{2}, D(u) \equiv d+3 B a_{1}^{A}(u)-3 B \nabla \varphi / 2$. Note that the Chern-Simon action are included in the total action during the transformations.

The Chern-Simon term with the derivatives $a^{V \prime}, a^{A \prime}$ eliminated is

$$
S_{C S}=-\mathcal{N} \frac{3}{2} B \int_{u_{c}}^{\infty} d u \frac{\left(a_{0}^{V}\left(j_{A}-\frac{3}{2} B \mu+3 B a_{0}^{V}\right)-f(u) D(u) a_{1}^{A}\right) \sqrt{\frac{1}{f(u)}+u^{3} x_{4}^{\prime 2}}}{\sqrt{f(u)\left(C(u)+D(u)^{2}\right)-\left(j_{A}-\frac{3}{2} B \mu+3 B a_{0}^{V}\right)^{2}}}
$$

From eq. (A.1), (A.2), (A.3), (2.5), and the boundary conditions, $a_{0}^{V}\left(u_{c}\right)=$ $\mu_{\text {source }}, a_{1}^{A}\left(u_{c}\right)=0$, we can solve to obtain the condition for the static equilibrium

$$
\left(x_{4}^{\prime}\left(u_{c}\right)\right)^{2}=\frac{1}{f_{c} u_{c}^{3}}\left[\frac{9}{d^{2}} \frac{\left(f_{c}\left(C_{c}+D_{c}^{2}\right)-\left(j_{A}-\frac{3}{2} B \mu+3 B a_{0}^{V}\left(u_{c}\right)\right)^{2}\right)}{\left(1+\frac{1}{2}\left(\frac{u_{T}}{u_{c}}\right)^{3}+3 n_{s} \sqrt{f_{c}}\right)^{2}}-1\right],
$$

where $f_{c} \equiv f\left(u_{c}\right), C_{c} \equiv C\left(u_{c}\right), D_{c} \equiv D\left(u_{c}\right)$.

Open Access. This article is distributed under the terms of the Creative Commons Attribution Noncommercial License which permits any noncommercial use, distribution, and reproduction in any medium, provided the original author(s) and source are credited.

\section{References}

[1] J.M. Maldacena, The large- $N$ limit of superconformal field theories and supergravity, Int. J. Theor. Phys. 38 (1999) 1113 [Adv. Theor. Math. Phys. 2 (1998) 231] [hep-th/9711200] [SPIRES].

[2] T. Sakai and S. Sugimoto, Low energy hadron physics in holographic QCD, Prog. Theor. Phys. 113 (2005) 843 [hep-th/0412141] [SPIRES]. 
[3] T. Sakai and S. Sugimoto, More on a holographic dual of $Q C D$, Prog. Theor. Phys. 114 (2005) 1083 [hep-th/0507073] [SPIRES].

[4] O. Aharony, J. Sonnenschein and S. Yankielowicz, A holographic model of deconfinement and chiral symmetry restoration, Annals Phys. 322 (2007) 1420 [hep-th/0604161] [SPIRES].

[5] A. Brandhuber, N. Itzhaki, J. Sonnenschein and S. Yankielowicz, Baryons from supergravity, JHEP 07 (1998) 020 [hep-th/9806158] [SPIRES].

[6] K. Ghoroku, M. Ishihara, A. Nakamura and F. Toyoda, Multi-quark baryons and color screening at finite temperature, Phys. Rev. D 79 (2009) 066009 [arXiv:0806.0195] [SPIRES].

[7] K. Ghoroku and M. Ishihara, Baryons with D5 Brane Vertex and k-Quarks, Phys. Rev. D 77 (2008) 086003 [arXiv:0801.4216] [SPIRES].

[8] M.V. Carlucci, F. Giannuzzi, G. Nardulli, M. Pellicoro and S. Stramaglia, AdS-QCD quark-antiquark potential, meson spectrum and tetraquarks, Eur. Phys. J. C 57 (2008) 569 [arXiv: 0711.2014] [SPIRES].

[9] W.-Y. Wen, Multi-quark potential from AdS/QCD, Int. J. Mod. Phys. A 23 (2008) 4533 [arXiv:0708.2123] [SPIRES].

[10] P. Burikham, A. Chatrabhuti and E. Hirunsirisawat, Exotic Multi-quark States in the Deconfined Phase from Gravity Dual Models, JHEP 05 (2009) 006 [arXiv:0811.0243] [SPIRES].

[11] O. Bergman, G. Lifschytz and M. Lippert, Holographic Nuclear Physics, JHEP 11 (2007) 056 [arXiv:0708.0326] [SPIRES].

[12] P. Burikham, E. Hirunsirisawat and S. Pinkanjanarod, Thermodynamic Properties of Holographic Multiquark and the Multiquark Star, JHEP 06 (2010) 040 [arXiv:1003.5470] [SPIRES].

[13] D.T. Son and M.A. Stephanov, Axial anomaly and magnetism of nuclear and quark matter, Phys. Rev. D 77 (2008) 014021 [arXiv:0710.1084] [SPIRES].

[14] O. Bergman, G. Lifschytz and M. Lippert, Magnetic properties of dense holographic QCD, Phys. Rev. D 79 (2009) 105024 [arXiv:0806.0366] [SPIRES].

[15] P. Burikham, Magnetic properties of holographic multiquarks in the quark-gluon plasma, JHEP 04 (2010) 045 [arXiv:0909.0614] [SPIRES].

[16] C.V. Johnson and A. Kundu, External Fields and Chiral Symmetry Breaking in the Sakai-Sugimoto Model, JHEP 12 (2008) 053 [arXiv:0803.0038] [SPIRES].

[17] F. Preis, A. Rebhan and A. Schmitt, Inverse magnetic catalysis in dense holographic matter, JHEP 03 (2011) 033 [arXiv: 1012.4785] [SPIRES].

[18] T. Vachaspati, Magnetic fields from cosmological phase transitions, Phys. Lett. B 265 (1991) 258 [SPIRES].

[19] D.E. Kharzeev, L.D. McLerran and H.J. Warringa, The effects of topological charge change in heavy ion collisions: 'Event by event P and CP-violation', Nucl. Phys. A 803 (2008) 227 [arXiv: 0711.0950] [SPIRES].

[20] R.C. Duncan and C. Thompson, Formation of very strongly magnetized neutron stars implications for gamma-ray bursts, Astrophys. J. 392 (1992) L9 [SPIRES]. 
[21] E. Witten, Baryons and branes in anti de Sitter space, JHEP 07 (1998) 006 [hep-th/9805112] [SPIRES].

[22] D.J. Gross and H. Ooguri, Aspects of large- $N$ gauge theory dynamics as seen by string theory, Phys. Rev. D 58 (1998) 106002 [hep-th/9805129] [SPIRES].

[23] O. Aharony, S.S. Gubser, J.M. Maldacena, H. Ooguri and Y. Oz, Large-N field theories, string theory and gravity, Phys. Rept. 323 (2000) 183 [hep-th/9905111] [SPIRES].

[24] O. Bergman, G. Lifschytz and M. Lippert, Response of Holographic QCD to Electric and Magnetic Fields, JHEP 05 (2008) 007 [arXiv: 0802.3720] [SPIRES].

[25] E.G. Thompson and D.T. Son, Magnetized baryonic matter in holographic QCD, Phys. Rev. D 78 (2008) 066007 [arXiv:0806.0367] [SPIRES].

[26] G. Lifschytz and M. Lippert, Holographic Magnetic Phase Transition, Phys. Rev. D 80 (2009) 066007 [arXiv:0906.3892] [SPIRES]. 